\title{
Resolution of Upper Mantle Structure Using Higher Modes of Rayleigh Waves (*).
}

\author{
L. KNOPOFF $(* *)-$ G. F. PANZA $(* * *)$
}

Received on January 9 th, 1978.

\begin{abstract}
Riassunto - Mediante il calcolo degli elementi diagonali della matrice degli errori del modello è stato possibile determinare il potere risolutivo di un certo insieme di dati rispetto ad alcuni dei parametri strutturali caratterizzanti il modello stesso. I dati considerati sono costituiti da un insieme discreto di velocità di fase per i primi sei modi delle onde di Rayleigh, a periodi facilmente ottenibili me. diante luso dei dati prodotti dalla WWSSN a lungo periodo. Per una struttura continentale formata da crosta, lid, canale, sottocanale e strato a spinello, le densità non possono essere determinate con una precisione migliore di $+0.25 \circ$ $+0.050 \mathrm{~g} / \mathrm{cm}^{3}$ anche facendo l'ipotesi che le densità siano parametri del modello non correlati. Inoltre, usando solo i dati relativi al modo fondamentale, le proprietà del lid non possono essere determinate con una precisione migliore di $15 \mathrm{~km}$ e di $0.08 \mathrm{~km} / \mathrm{sec}$ per quel che riguarda lo spessore e la velocità delle onde $S$, rispettivamente; l'aggiunta di dati di dispersione relativi ai modi superiori non apporta alcun miglioramento. Le proprietà degli strati più profondi, invece, sono risolte meglio quando è possibile impiegare anche $\mathrm{i}$ dati di dispersione relativi ai modi superiori.
\end{abstract}

AnSTRACT - Through the computation of the diagonal elements of the model error matrix the resolving power of a hypothetical data set has been investigated with respect to a number of structural parameters. The «data » are a discrete set of phase velocities for the first six Rayleigh modes at periods easily obtainable from the use of WWSSN long period seismograms. In the case of a continental structure made up of crust, lid, channel, subchannel and spinel layers, densities cannot be resolved to better than +0.25 to $+0.3 \mathrm{~g} / \mathrm{cm}^{3}$. even with the assumption

(*) Publication number 1779. Institute of Geophysics and Planetary Physics, University of California, Los Angeles, and Publication number 74, P. F. Geodinamica, CNR, Rome.

$\left.{ }^{* * *}\right)$ Institute of Geophysics and Planetary Physics, University of California, Los Angeles.

(*:*:) Istituto di Geodesia e Geofisica, Università di Bari. Bari, Italy. 
that densities are uncorrelated model parameters. Furthermore, the properties of the lid can be resolved to no better than $15 \mathrm{~km}$ in thickness and $0.08 \mathrm{~km} / \mathrm{sec}$ in velocity using only fundamental mode. The addition of higher mode data does not represent an improvement; deeper layers are better resolved if higher mode data are considered.

\section{INTRODUCTION.}

The World-Wide Standard Seismographic Network (WWSSN) provided significant impetus to the study of upper mantle cross-sections by making possible the recording of seismic surface waves at wavelengths of the order of several hundreds of kilometers on a relatively densely spaced. array of similar instruments. Most of the applications of analysis of such recordings have been made mainly through the study of fundamental mode Rayleigh wave dispersion which has led to a considerable elaboration of the lateral inhomogeneities in the earth's upper mantle (Knopoff, 1972). However, limitations of band width of the instrumentation have made it difficult to record surface wave signals routinely with periods greater than $150 \mathrm{sec}$; this feature, in turn limits the depth to which exploration of the $S$-wave structure of the upper mantle can be made to about 200 to $250 \mathrm{~km}$, using fundamental mode Rayleigh waves. The recording of 4 th or 5 th order higher modes to periods as great as 50 or $60 \mathrm{sec}$ permits sampling of the mantle to much greater depths, since the eigenfunctions of these modes at these periods are significantly nonzero to depths as great as $1000 \mathrm{~km}$. Thus the instruments of the WWSSN and similar instruments can be used in a regional exploration of possible inhomogeneities to much greater depths than hitherto. However the analysis of recordings of higher mode surface waves in the presence of a strong fundamental, requires the use of arrays of long-period seismographs, a subject which has received attention in recent years (Nolet, 1975, 1977; Nolet and Panza, 1976; Mitchel, 1977; Panza and Scalera, 1978). In this paper we are concerned, not with the data analysis to extract information at the higher modes, but rather with the improvement in the resolution of structural parameters to be obtained by adding phase velocity data in the higher modes. 


\section{Continental structure.}

We attack this problem in a manner similar to that considered by Knopoff and Chang (1977). We start with a presumed known continental structure. The forward problem for the generation of phase velocities in all modes is then solved exactly. These dispersion curves are then digitized to form a "basic data set ". To the data set we add random noise in the amount $\sigma(T ; n)$ where $T$ is the period and $n$ is the mode number. The quantity $\sigma$ is the standard deviation in $\mathrm{km} / \mathrm{sec}$ of the phase velocity that we imagine might be measured at the particular period and mode number. If the standard errors are small enough, the span of models, that yield dispersion curves whose rms differences from the reference curve are less than $\sigma$, is sufficiently small that the problem is a locally linear one. In the linear inverse problem the partial derivatives of phase velocity with respect to model parameters are constants over the entire span of models that are acceptable within the rms criterion $\sigma$.

In order to isolate the ability of a given data set to provide resolution of particular aspects of internal structure of the earth, we restrict the problem to exclude other effects which we consider to be tangential to the main purpose of this study. Therefore we do not consider effects of sphericity and gravity on the propagation of surface waves. For our purposes, we consider wave propagation in an ideal, horizontally-layered structure and consider perturbations of this structure. In the case of linearity of the inversion, we may assume that gravity, curvature and other effects will modify the starting structure and the perturbed structures equally. Furthermore we do not have to be as faithful as would be otherwise required to simulate the exact nature of the mantle of the earth. Our purpose is to understand the ability of a hypothetical data set to resolve structural parameters in a hypothetical earth model.

The starting model that was chosen is given in Table I and is the same assumed by Knopoff and Chang, (1977). It consists of a crust $50 \mathrm{~km}$ in thickness, overlying an upper mantle consisting of a lid, a low- 
Table 1. Crust - Upper Mantle Structure for Continent.

\begin{tabular}{|c|c|c|c|c|c|}
\hline & Depth $(\mathrm{km})$ & Thickness $(\mathrm{km})$ & $\beta(\mathrm{km} / \mathrm{sec})$ & $\alpha(\mathrm{km} / \mathrm{sec})$ & $\rho\left(\mathrm{g} / \mathrm{cm}^{3}\right)$ \\
\hline \multirow{5}{*}{ CRUST } & 0 & & & & \\
\hline & 10 & 10 & 3.49 & 6.05 & 2.75 \\
\hline & & 20 & 3.67 & 6.35 & 2.85 \\
\hline & 30 & & & & \\
\hline & & 20 & 3.85 & 7.05 & 3.08 \\
\hline LID & \multirow{2}{*}{115} & 65 & 4.65 & 8.17 & 3.45 \\
\hline CHANNEL & & 250 & 4.30 & 8.55 & 3.54 \\
\hline SUBCHANNEL & \multirow{2}{*}{450} & 85 & 4.75 & 8.80 & 3.65 \\
\hline SPINEL LAYER & & 200 & 5.30 & 9.80 & 3.98 \\
\hline \multirow[t]{3}{*}{ POST-SPINEL LAYER } & 050 & 400 & 6.20 & 11.15 & 4.43 \\
\hline & 1290 & 240 & 6.48 & 11.78 & 4.63 \\
\hline & & $\infty$ & 6.62 & 12.02 & 4.71 \\
\hline
\end{tabular}


velocity channel for $S$-waves, a subchannnel, and phase transitions at 450 and $650 \mathrm{~km}$. The deeper mantle is replaced by a half-space starting at $1290 \mathrm{~km}$ depth. The phase velocities for the first six Rayleigh modes in this flat earth model are shown in Figure 1. In our notation, mode 0 is the fundamental mode, mode 1 is the first higher mode, etc.. Each of these curves has been assumed to have been digitized at the periods indicated in Table 2. These period ranges are not inconsistent with those obtained in the recent explorations of stacking procedures to isolate the higher modes of Rayleigh waves cited above. The values in parentheses

TABLE 2

\begin{tabular}{llllllllll}
\hline MODE & \multicolumn{8}{c}{ PERIOD RANGE (sec) } & N \\
\hline 0 & 20 & $(5)$ & 40 & $(10)$ & 100 & $(25)$ & 250 & 17 \\
$i$ & 20 & $(5)$ & 40 & $(10)$ & 100 & $(25)$ & 150 & 13 \\
2 & 20 & $(5)$ & 40 & $(10)$ & 100 & & & 11 \\
3 & 20 & $(5)$ & 40 & $(10)$ & 60 & & 7 \\
4 & 20 & $(5)$ & 40 & $(10)$ & 50 & & & 6 \\
5 & 20 & $(5)$ & 40 & & & & & 5 \\
\hline
\end{tabular}

represent the period interval between adjacent "digitized values ". The number of digitized values in each mode is also indicated.

To embark on the inversion, we assume that the value of $\sigma$ is uniformly $0.03 \mathrm{~km} / \mathrm{sec}$ for all values. In the inversion, we take this to mean that a model will be acceptable if the rms difference between the phase velocities for the reference model and the test model is less than $\sigma$ or some small multiple thereof. We use as our measure of resolution that considered by Knopoff and Chang (1977), namely the diagonal elements of the model error matrix

$$
\left\{\frac{1}{N} \sum_{i, n}\left(\frac{\partial c\left(T_{i}, n\right)}{\partial P_{i}} \frac{n}{2}\right)^{2}\left[\sigma\left(T_{i}, n\right)\right]^{-2}\right\}^{-1 / 2}
$$

which are the intercepts of the solution ellipsoid with the parameter axes, $P_{j}$. 


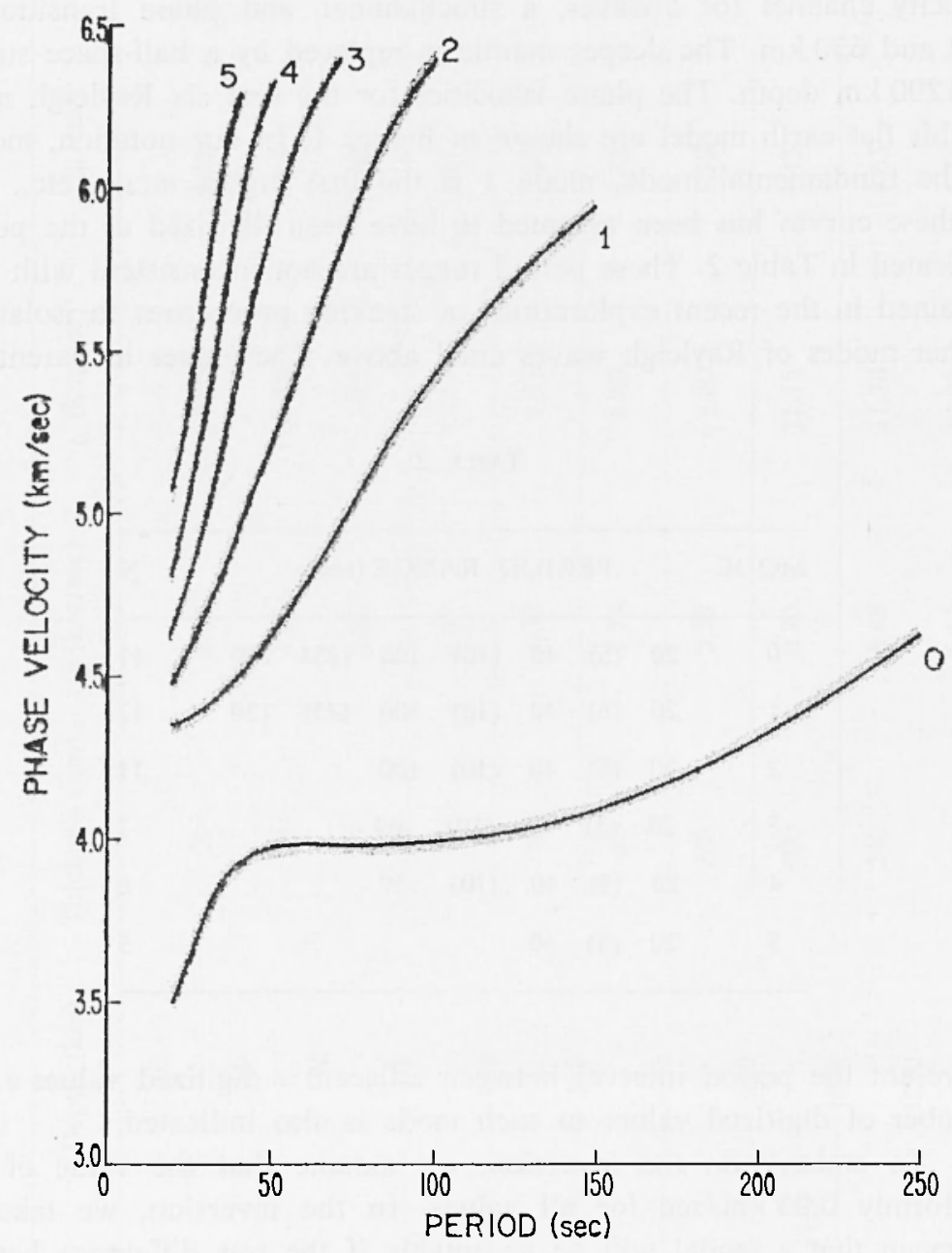

Fig. 1. Dispersion curves for the first six Rayleigh modes for continental model. 0 indicates the fundamental mode, 1 indicates the first higher mode, and so on. The shaded region corresponds to $\sigma=0.03 \mathrm{~km} / \mathrm{sec}$.

In this example, we consider the ability of the data set, or parts of the data set, to resolve thirteen different structural parameters. These are the thickness, $S$-wave velocity and density of the lid, channel, subchannel and "spinel " layers. In addition we investigate the ability of the fundamental mode data set to resolve the crustal thickness; in 
this case we have assumed the three individual layers of the crust always are found in the same ratio as they appear in the original model. We have introduced the possibility of studying the density resolution of higher modes, since the higher modes are reputed to be more sensitive to density influences than is the fundamental mode. The results of the analysis for the resolution parameter (1) are listed in Tables 3-5. In the upper parts of each table we have listed the results of the calculation for each of the modal phase velocity curves taken separately. In the lower parts of the tables we indicate the effects of increasing the data sets by including more and more data, starting with the fundamental mode and adding successively the higher modes in order. In the case of layer thickness analysis, the fundamental mode data are able to provide information about crustal thickness to within $41 / 2 \mathrm{~km}$ at the $1 \sigma$ level. We further see that the fundamental mode data do the best job in resolving the thickness and $S$-wave velocity of the lid, that the third higher mode is best for determining channel thickness and that the fifth higher mode is best for determining the subchannel and spinel layer thicknesses and the $S$-wave velocity in the spinel layer. In fact, additional data in even higher modes might be more appropriate to resolve these layer thicknesses and velocity but we have not carried the calculations out that far, nor do we see any need to, in view of present capabilities of data analysis. The $S$-wave velocity in the channel is best resolved by the first higher mode data and the subchannel velocity is best given by the third higher mode. In no case does the data set we have described give good resolution of density in the upper mantle. Although the ability to resolve layer thickness to within 30 or $40 \mathrm{~km}$ may not seem useful to practitioners experienced in other areas of seismic data analysis, nevertheless, we believe that such results present an accurate, if unflatteringly realistic picture of what can be done with surface wave studies.

When higher modes are added to the data set, the ability to resolve those parameters that are well resolved with fundamental mode data under criterion (1) decreases. This is not unexpected because of the rms nature of the criterion. In most typical uses of least squares criteria, the addition of irrelevant data to a data set weakens the quality of a parametric fit. Since most modern linear inversions depend on one form or another of a least squares fit to the data, this result suggests that perhaps a more careful selection of the data would lead to a better resolution of model parameters than simply an indiscriminate 
TABLE 3. Layer Thickness Resolution.

$$
(\mathrm{Km})
$$

\begin{tabular}{l|cccccc}
\hline EARTH \ MODE & 0 & 1 & 2 & 3 & 4 & 5 \\
\hline CRUST & 4.55 & - & - & - & - & - \\
LID & 30.9 & 138. & 59.6 & 38.2 & 40.3 & 52.0 \\
CHANNEL & 49.2 & 113. & 56.9 & 44.5 & 48.1 & 60.5 \\
SUBCHANNEL & 48.8 & 39.2 & 30.6 & 29.3 & 28.4 & 28.1 \\
«SPINEL » LAYER & 66.4 & 45.8 & 39.3 & 33.0 & 30.3 & 26.6 \\
\hline EARTH, MODES & $0 !$ & 012 & 0123 & 01234 & 012345 & \\
\hline LID & & & & & & \\
CHANNEL & 38.3 & 40.8 & 40.1 & 40.1 & 40.7 & \\
SUBCHANNEL & 54.0 & 52.5 & 50.9 & 50.4 & 50.8 & \\
«SPINEL » LAYER & 43.1 & 37.8 & 35.7 & 34.4 & 33.4 & \\
\hline
\end{tabular}

rush to acquire more and more data in the hope that this would improve resolution. Linder a least squares fit this criterion is not assured.

Suppose that we inquire into the most suitable data set for the determination of any given model parameter. If we assume that our premise is correct, namely that the inclusion of too much data is deleterious to the program of optimizing resolution, then we may approach the prcblem of finding the subset of data for our purpose by the following reasoning. Let us start with a large data set, which from Tables 3 to 5, seemingiy does not do as good a job as a smaller data set, and we start to reject those data which make the largest contributions to the estimate of (1). For example, from Table 3 , if our intention is to provide the data set that minimizes the resolution for the layer thickness of the lid in the upper mantle structure of Table I, we should discard all the higher 
TABle 4. Shear Wave Velocity Resolution.

$(\mathrm{Km} / \mathrm{sec})$

\begin{tabular}{|c|c|c|c|c|c|c|}
\hline EARTH /MODE & 0 & 1 & 2 & 3 & 4 & 5 \\
\hline LID & 0.145 & 0.526 & 0.227 & 0.168 & 0.195 & 0.271 \\
\hline CHANNEL & 0.070 & 0.037 & 0.045 & 0.055 & 0.054 & 0.057 \\
\hline SUBCHANNEL & 0.371 & 0.211 & 0.154 & 0.136 & 0.145 & 0.158 \\
\hline «SPINEL » LAYER & 0.329 & 0.147 & 0.118 & 0.099 & 0.085 & 0.077 \\
\hline EARTH $/$ MODES & $0 !$ & 012 & 0123 & 01234 & 012345 & \\
\hline LID & 0.187 & 0.195 & 0.190 & 0.190 & 0.195 & \\
\hline CHANNEL & 0.048 & 0.047 & 0.048 & 0.049 & 0.049 & \\
\hline SUBCHANNEL & 0.269 & 0.216 & 0.193 & 0.186 & 0.183 & \\
\hline "SPINEL » L $\triangle Y E R$ & 0.199 & 0.163 & 0.144 & 0.131 & 0.122 & \\
\hline
\end{tabular}

mode data and retain only the fundamental mode data, since the value of $30.9 \mathrm{~km}$ is less than 38.3, etc. for all the cases of higher modes combined with the fundamental. But if it is valid that we should reject the data from all the modal branches but one, why should we not reject all the most irrelevant data from within a given modal branch except the most relevant datum? This logic, suggests to us that maximum resolution for a given model parameter is achieved by retaining only one datum, specifically that for which

$$
\left(\frac{\partial c\left(T_{i}, n\right)}{\partial P_{j}}\right)^{-1} \sigma\left(T_{i}, n\right)
$$


TABLE 5. Density Resolution.

$\left(\mathrm{g} / \mathrm{cm}^{3}\right)$

\begin{tabular}{l|cccccc}
\hline EARTH \MODE & 0 & 1 & 2 & 3 & 4 & 5 \\
\hline LID & 0.361 & 1.62 & 0.815 & 0.665 & 0.745 & 1.52 \\
CHANNEL & 0.399 & 0.497 & 0.798 & 0.909 & 1.03 & 1.24 \\
SUBCHANNEL & 0.885 & 0.668 & 0.648 & 0.733 & 0.736 & 1.02 \\
«SPINEL » LAYER & 0.706 & 0.636 & 0.595 & 0.777 & 1.15 & 1.59 \\
\hline EARTH MODES & $0 !$ & 012 & 0123 & 01234 & 012345 & \\
\hline LID & & & & & & \\
CHANNEL & 0.471 & 0.519 & 0.537 & 0.551 & 0.573 & \\
SUBCHANNEL & 0.434 & 0.482 & 0.513 & 0.535 & 0.554 & \\
«SPINEL 》 LAYER & 0.768 & 0.730 & 0.730 & 0.731 & 0.746 \\
\hline
\end{tabular}

is a minimum. In Tables 6 to 8 we have listed the resolution to be expected under criterion (2) for each of the modal data set branches taken scparately, together with the period, in parentheses, at which the extremum occurs. Finally we have indicated in the table, by enclosure, the single entry that performs the optimum resolution, i. e. that cntry out of all the 59 data that minimizes (2). Thus, for example, if only the datum of fundamental mode Rayleigh wave phase velocity at a pericd of $40 \mathrm{sec}$ were measured and used in the inversion, the thickness of the lid could be better determined than with any other datum or combination of data, under this assumption of the values of $\sigma$. Of principal curiosity is the fact that no data from higher mode 2 and virtually none from modes 3 or 4 enter into the optimization of resolution for any of the model parameters. 
TABIE 6. Layer Thickness Resolution.

$(\mathrm{km})$

\begin{tabular}{|c|c|c|c|c|c|c|}
\hline EARTH \MODE & 0 & 1 & 2 & 3 & 4 & 5 \\
\hline CRUST & 3.30 & - & - & - & - & - \\
\hline LID & $14.0(40)$ & $36.9(60)$ & $24.5(25)$ & $18.7(20)$ & $22.0(40)$ & $21.1(20)$ \\
\hline CHANNEL & $35.7(125)$ & $32.7(20)$ & $31.5(20)$ & $30.6(30)$ & $29.4(25)$ & $26.2(20)$ \\
\hline SUBCHANNELL & $26.2(225)$ & $24.9(60)$ & $28.0(80)$ & $25.7(50)$ & $23.7(35)$ & $24.5(25)$ \\
\hline «SPINEL 》 LAYER & $23.8(250)$ & $23.3(90)$ & $22.4(100)$ & $24.6(60)$ & $21.7(40)$ & $20.5(25)$ \\
\hline
\end{tabular}


TABLE 7. Shear Wave Velocity Resolution.

$(\mathrm{km} / \mathrm{sec})$

\begin{tabular}{l|cccccc}
\hline MODE \EARTH & 0 & 1 & 2 & 3 & 4 & 5 \\
\hline LID & $0.077(40)$ & $0.350(60)$ & $0.198(30)$ & $0.114(20)$ & $0.142(40)$ & $0.221(25)$ \\
CHANNEL & $0.047(150)$ & $0.051(20)$ & $0.035(20)$ & $0.043(30)$ & $0.042(25)$ & $0.040(20)$ \\
SUBCHANNELL & $0.167(250)$ & $0.124(70)$ & $0.112(40)$ & $0.103(60)$ & $0.115(25)$ & $0.103(20)$ \\
«SPINEL 》 LAYER & $0.118(250)$ & $0.079(100)$ & $0.073(60)$ & $0.069(40)$ & $0.060(35)$ & $0.056(25)$ \\
\hline
\end{tabular}


A casual assessment of the above discussion would lead the reader to the tantalizing, but erroneous conclusion that the determination of optimum values for, let us say, $N$ mantle parameters (and their uncertainties) is best accomplished by taking only $N$ measurements at certain selected mode-frequency pairs and ignoring all other measurements. The fallacy in this statement arises becasuse the curves of partial derivatives of phase velocity are not delta functions. The statement (2) illustrated by tables $6-8$, is only valid if the partial derivative curves are all delta functions, which they are not, as is well known from linear inverse theory (Backus and Gilbert, 1968). Another way of stating this is, that the conclusions represented by criterion (2) are valid if all other parameters are fixed i. e. known, during the exploration for the optimum value of the jth parameter. But since none of the $N$ parameters is known we must take into account the feature that the partial derivative curves often have large wings remcte from the peaks. In this case, the variation of values of phase velocities with respect to one parameter in the neighborhood of, let us say a peak in the partial derivative curve, influences the value of the variation in phase velocity in the neighborhood of a partial derivative with respect to another parameter. The worst possible case of two coupled parameters occurs when they both have a peak at the same period-mode number; a case in point arises in the effort to determine both the velocity and thickness of the lid; the partial derivative curves for the two parameters both have their peaks at $40 \mathrm{sec}$ for the fundamental mode. Thus these two parameters are not independent under criterion (2) and the resolution of each is certainly poorer than the values quoted in Tables 6 and 7, although they are probably better than the values indicated in Tables 3 and 4 . The full problem requires the determination of the period-mode pairs for which the quantities $\delta P_{i}$ are minima subject to the condition,

$$
\sum_{j} \frac{\partial c\left(T_{i}, n\right)}{\partial P_{i}} \delta P_{j}=\sigma\left(T_{i}, n\right)
$$

This is a linear programming problem of some complexity. It can be shown that often less than $N$ solutions to the problem exist in the case of strongly correlated model parameters. A fuller discussion of the aspects of the optimization problem in connection with criterion (3) will be presented elsewhere. 
Table 8. Density Resolution.

$\left(\mathrm{g} / \mathrm{cm}^{3}\right)$

\begin{tabular}{l||cccccc}
\hline EARTH & 0 & 1 & 2 & 3 & 4 & 5 \\
\hline LID & $0.261(225)$ & $0.882(50)$ & $0.477(40)$ & $0.406(50)$ & $0.523(40)$ & $0.990(40)$ \\
CHANNEL & $0.264(100)$ & $0.249(150)$ & $0.473(35)$ & $0.397(45)$ & $0.508(25)$ & $0.586(30)$ \\
SLBCHANNEL & $0.412(225)$ & $0.313(100)$ & $0.357(80)$ & $0.420(50)$ & $0.578(25)$ & $0.602(25)$ \\
«SPINEL » LAYER & $0.259(250)$ & $0.279(150)$ & $0.278(70)$ & $0.487(60)$ & $0.575(35)$ & $0.863(25)$ \\
\hline
\end{tabular}




\section{Conclusion.}

We conclude that, in an absolute sense, densities cannot be resolved to better than \pm 0.25 or $\pm 0.3 \mathrm{gm} / \mathrm{cm}^{3}$ with the use of higher mode data up to the 5 th order, even with the assumption that densities are uncorrelated model parameters. However, to determine the properties of the lid, fundamental mode data probably suffice to give resolution to no better than $15 \mathrm{~km}$ in thickness and $0.08 \mathrm{~km} / \mathrm{sec}$ in velocity; the actual case is worse than this since the variables are correlated. In the case of the lid, addition of nigher mode data probably does not represent an improvement. These estimates, and in general those of tables 6-8, probably represent optimal bounds on the resolution; the actual values depend on the correlation of the model parameters used in the inversion.

\section{REFERENCES}

Backus, G. and Gildert, J. F., 1968. - The Resolving Power of Gross Earth Data. Geophys. J. Roy. Astron. Soc., 16, 169-205.

KnopofF, L., 1972. - Observation and Inversion of Surface Wave Dispersion. Tectonophysics, 13, 497-519.

Knopoff, L. and Chang, F. S., 1977. - The Inversion of Surface Wave Dispersion Data with Random Errors. J. Geophys., 43, 299-310.

Mitchel, R. G., 1977. - The Structure of Western North America from Multimode Rayleigh Wave Dispersion. Ph. D. Thesis, U. C. L. A.

Nolet, G., 1975. - Higher Rayleigh Modes in Western Europe. Geophys. Res. Letters, 2, 60-62.

NoLet, G., 1977. - The Upper Mantle under Western Europe inferred from the Dispersion of Rayleigh Modes. J. Geophys., 43. 265-286.

Nolet, G. and Panza, G. F., 1976. - Array Analysis of Seismic Surlace Waves: Limits and Possibilities. Pure Appl. Geophys., 114, 775-790.

Panza, G. F. and Scalera, G., 1978. - Higher Modes Dispersion Measurements. Pure Appl. Geophys., 116. 1274-1283. 